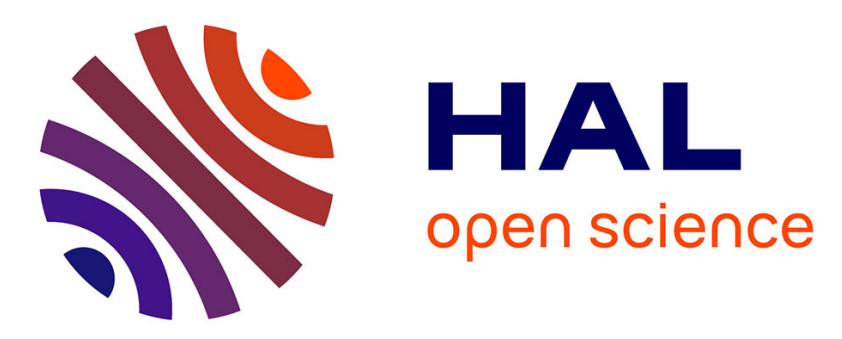

\title{
The plastoquinone pool outside the thylakoid membrane serves in plant photoprotection as a reservoir of singlet oxygen scavengers
}

\author{
Brigitte Ksas, Bertrand Legeret, Ursula Ferretti, Anne Chevalier, Pavel \\ Pospíšil, Jean Alric, Michel Havaux
}

\section{To cite this version:}

Brigitte Ksas, Bertrand Legeret, Ursula Ferretti, Anne Chevalier, Pavel Pospíšil, et al.. The plastoquinone pool outside the thylakoid membrane serves in plant photoprotection as a reservoir of singlet oxygen scavengers. Plant, Cell and Environment, 2018, 41 (10), pp.2277-2287. 10.1111/pce.13202 . hal-01946443

\section{HAL Id: hal-01946443 \\ https://hal.science/hal-01946443}

Submitted on 8 Jan 2019

HAL is a multi-disciplinary open access archive for the deposit and dissemination of scientific research documents, whether they are published or not. The documents may come from teaching and research institutions in France or abroad, or from public or private research centers.
L'archive ouverte pluridisciplinaire HAL, est destinée au dépôt et à la diffusion de documents scientifiques de niveau recherche, publiés ou non, émanant des établissements d'enseignement et de recherche français ou étrangers, des laboratoires publics ou privés. 
2

3

4

5

6

7

8

9

10

\section{The plastoquinone pool outside the thylakoid membrane serves in plant photoprotection as a reservoir of singlet oxygen scavengers}

Brigitte Ksas ${ }^{1}$, Bertrand Legeret ${ }^{2}$, Ursula Ferretti ${ }^{3}$, Anne Chevalier ${ }^{1}$, Pavel Pospíši ${ }^{3}$, Jean Alric ${ }^{1}$, Michel Havaux ${ }^{1}$

${ }^{1}$ CEA Cadarache, CNRS UMR 7265 Biologie Végétale et Microbiologie Environnementales, Aix Marseille Université, Laboratoire d'Ecophysiologie Moléculaire des Plantes, 13108 Saint-Paul-lez-Durance, France

${ }^{2}$ CEA Cadarache, CNRS UMR 7265 Biologie Végétale et Microbiologie Environnementales, Aix Marseille Université, Laboratoire de Bioénergétique et Biotechnologie des Bactéries et Microalgues, 13108 Saint-Paul-lez-Durance, France

${ }^{3}$ Department of Biophysics, Centre of the Region Haná for Biotechnological and Agricultural Research, Faculty of Science, Palacký University, Šlechtitelů 27, 783 71, Olomouc, Czech Republic.

\section{Correspondence}

Michel Havaux. Email: michel.havaux@cea.fr

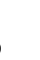




\section{Abstract}

The Arabidopsis vte1 mutant is devoid of tocopherol and plastochromanol (PC-8). When exposed to excess light energy, vte1 produced more singlet oxygen $\left({ }^{1} \mathrm{O}_{2}\right)$ and suffered from extensive oxidative damage compared to the wild type. Here we show that overexpressing the SOLANESYL DIPHOSPHATE SYNTHASE 1 (SPS1) gene in vte1 induced a marked accumulation of plastoquinone (PQ-9) and rendered the vte1 SPS1oex plants tolerant to photooxidative stress, indicating that PQ-9 can replace tocopherol and PC-8 in photoprotection. High PQ-9 levels were associated with a noticeable decrease in ${ }^{1} \mathrm{O}_{2}$ production and with higher levels of PQ-C, a ${ }^{1} \mathrm{O}_{2}$-specific PQ-9 oxidation product. The extra PQ-9 molecules in the vte1 SPS1oex plants were stored in the plastoglobules and the chloroplast envelopes, rather than in the thylakoid membranes, whereas PQ-C was found almost exclusively in the thylakoid membranes. Upon exposure of wild-type plants to high light, the thylakoid PQ-9 pool decreased, while the extra-thylakoid pool remained unchanged. In vte1 and vte1 SPS1oex plants, the PQ-9 losses in high light were strongly amplified, affecting also the extra-thylakoid pool, and PQ-C was found in high amounts in the thylakoids. We conclude that the thylakoid PQ-9 pool acts as a ${ }^{1} \mathrm{O}_{2}$ scavenger and is replenished from the extra-thylakoid stock.

\section{KEYWORDS}

plastoquinone, antioxidant, reactive oxygen species, photoprotection, tocopherol

\section{INTRODUCTION}

Plastoquinone-9 (PQ-9) is essentially viewed as a component of the photosynthetic electron transport chain in chloroplasts, carrying electrons from photosystem II (PSII) to cytochrome b6/f (Amesz, 1973). It is thus essential for the photosynthetic activity of plants, and mutations inhibiting PQ-9 accumulation led to a lethal phenotype in Arabidopsis and maize (Cook \& Miles, 1992; Norris, Barrette, \& DellaPenna, 1995). Through its redox state, the PQ-9 pool can also play a role in the regulation of gene expressions and enzyme activities (Pfannschmidt et al., 2009; Rochaix, 2014). However, several observations obtained in recent in vitro and in vivo studies have raised the idea that the physiological role of PQ-9 can be more diverse than just electron shuttling from PSII to PSI. In particular, in vitro studies have revealed that PQ-9 has antioxidant properties, being able to quench singlet oxygen $\left({ }^{1} \mathrm{O}_{2}\right)$ and to inhibit oxidation of lipid membranes (Gruszka, Pawlak, \& Kruk, 2008; Nowicka, Gruszka, \& Kruk, 2013; Yadav, Kruk, Sinha, \& Pospisil, 2010). In isolated thylakoids, maintenance of the PQ-9 pool in the reduced state with an electron transport inhibitor was associated with a lowering of high light-induced lipid 
peroxidation, suggesting an antioxidant role for reduced PQ-9 (Hundal, Forsmark-Andrée, Ernster, \& Andersson, 1995).

Tocopherol and plastoquinone are synthesized through a common pathway until homogentisate (HGA) where SOLANESYL DIPHOSPHATE SYNTHASE 1 (SPS1) introduces the bifurcation towards PQ-9 and PC-8. Accumulation of PQ-9 in leaves by overexpression of the SPS1 gene in SPS1oex Arabidopsis plants reduced lipid peroxidation and increased the tolerance to photooxidative stress (Ksas, Becuwe, Chevalier, \& Havaux, 2015). However, because the SPS1oex plants also accumulated plastochromanol-8 (PC-8), a PQ-9 metabolite with known antioxidative activities (Kruk, Szymanska, Cela, \& Munne-Bosch, 2014), photoprotection could not be unambiguously and exclusively attributed to PQ-9. Nevertheless, sudden exposure of Arabidopsis plants to high light stress resulted in a marked loss of PQ-9 (Ksas et al., 2015) and to an accumulation of oxidized derivatives formed by the interaction between PQ-9 and ${ }^{1} \mathrm{O}_{2}$ (Szymanska \& Kruk, 2010), supporting the idea that $\mathrm{PQ}-9$ can act as a ${ }^{1} \mathrm{O}_{2}$ scavenger in vivo. Similarly, in the green microalga Chlamydomonas, PQ-9 was observed to decrease in high light when its re-synthesis was blocked by an inhibitor of hydroxylphenylpyruvate dioxygenase (Kruk \& Trebst, 2008).

In this context, an interesting observation was the various localizations of PQ-9 in thylakoid membranes, plastoglobules (Lichtenthaler \& Weinert, 1970; Zbierzak et al., 2009) and chloroplast envelopes (Soll, Schultz, Joyard, Douce, \& Block, 1985), suggesting that PQ-9 could play diverse roles in the chloroplasts. To investigate these roles, we have crossed the vte1 Arabidopsis mutant, deficient in both tocopherols and PC-8 (Porfiriva, Bergmuller, Tropf, Lemke, \& Dörmann, 2002), with SPS1oex lines previously described (Ksas et al., 2010) to generate vte1 SPS1oex plants that selectively accumulate PQ-9 in the absence of PC-8. The responses of vte1 SPS1oex plants to high light together with the analyses of PQ-9 localization and concentration demonstrate that PQ-9 is a ${ }^{1} \mathrm{O}_{2}$-scavenging antioxidant in planta and show the participation of the extra-thylakoid PQ-9 pool in this process through delivery of PQ-9 to the thylakoids in order to replace oxidized PQ-9 molecules.

\section{MATERIAL AND METHODS}

\subsection{Plant material and growth conditions}

Arabidopsis plants (Arabidopsis thaliana, Columbia-0 ecotype) were grown in a phytotron under controlled conditions of light $\left(150 \mu \mathrm{mol}\right.$ photons $\mathrm{m}^{-2} \mathrm{~s}^{-1}$, photoperiod $\left.8 \mathrm{~h}\right)$, temperature $\left(20^{\circ} \mathrm{C} / 18^{\circ} \mathrm{C}\right.$, day/night) and relative air humidity (60\%). Several mutant/transformed plants were examined in this work: the tocopherol cyclase mutant vte1 deficient in tocopherols (Porfirova et al., 2002), SPS1overexpressing lines described in a previous work (lines \#12 and \#14 in Ksas et al. (2015)) and a crossing between them (vte1 SPS1oex). Selection of homozygous vte1 SPS1oex plants was achieved first by 
screening the progeny for resistance to the herbicide BASTA and then by screening for total absence of tocopherol and PC-8 by HPLC analyses (see below for HPLC method). Photooxidative stress was induced by transferring plants to a growth chamber at $7{ }^{\circ} \mathrm{C} / 15^{\circ} \mathrm{C}$ (day/night, air temperature) under a PFD of $1500 \mu \mathrm{mol}$ photons $\mathrm{m}^{-2} \mathrm{~s}^{-1}$ and a photoperiod of $8 \mathrm{~h}$, as previously explained (Ksas et al., 2015). Depending of the experiment, the duration of the treatment was $48 \mathrm{~h}$ (photooxidative stress experiments) or $8 \mathrm{~h}$ (dynamics of the PQ-9 pools), as indicated in the legend of the figures.

\subsection{Prenyl lipid determinations}

Leaf discs ( 3 discs, diameter $1 \mathrm{~cm}$ ) were grinded in ethyl acetate. After centrifugation, the surpernatant was filtered and evaporated on ice under a stream of $\mathrm{N}_{2}$. The residue was recovered in methanol/hexane (17/1) and analyzed by HPLC, as described elsewhere (Ksas et al., 2015; Szymanska, Nowicka, \& Kruk, 2014; Kruk \& Karpinski, 2006). The column was a Phenomenex Kinetex $2.6 \mu$ m, 100 $x 4.6 \mathrm{~mm}, 100 \mathrm{~A}$. Separation of tocopherols, plastoquinone-9 and plastochromanol-8 was done in the isocratic mode with methanol/hexane (17/1) as solvent system and a flow rate of $0.8 \mathrm{ml} \mathrm{min}{ }^{-1}$. All prenyl lipids, except oxidized plastoquinone-9, were detected by their fluorescence at $330 \mathrm{~nm}$ with an excitation at $290 \mathrm{~nm}$. Plastoquinone-9 in the oxidized state was measured by its absorbance at 255 nm. Typical chromatograms are shown in Supplemental Fig. S1. Plastochromanol-8 and plastoquinone-9 standards were a kind gift from Dr. J. Kruk. Tocopherol standards were purchased from Sigma. Unless specified otherwise, the PQ-9 data given in this article correspond to the total content (oxidized $\mathrm{PQ}-9+$ reduced $\mathrm{PQH}_{2}-9$ ).

Determination of the photoactive and non-active fractions of plastoquinone-9 was done following the protocol described in Kruk \& Karpinski (2006) modified by Ksas et al. (2015). Leaf samples were frozen in liquid nitrogen as quickly as possible to avoid changes in the plastoquinone redox state during sample preparation. Two discs (diameter, $0.8 \mathrm{~cm}$ ) were punched out from each leaf. To determine the pool of photoactive plastoquinone-9, we first measured the amount of reduced plastoquinone-9 (plastoquinol-9) in one disc after a dark-adaptation period of $2 \mathrm{~h}$. The dark-adapted samples were frozen in liquid nitrogen after 3-s illumination with far-red light to ensure full oxidation of the photoactive pool of plastoquinone. Then, plastoquinol-9 was re-quantified in the discthat had been exposed to a high PFD of white light $\left(2000 \mu \mathrm{mol}\right.$ photons $\left.\mathrm{m}^{-2} \mathrm{~s}^{-1}\right)$ for $15 \mathrm{~s}$. Illumination was done in a mortar that was filled with liquid nitrogen after the 15-s illumination in order to freeze the samples in the light. The size of the photoactive pool was calculated as the difference between the plastoquinol9 content in the light and the plastoquinol-9 content in the dark. The size of the non-photoactive pool of plastoquinone-9 was the sum of the amount of plastoquinol-9 in the dark (not reoxidable in the dark) and the amount of oxidized plastoquinone- 9 in the light (not reducible by high light). This pool 
corresponds mainly to the plastoquinone molecules present in the plastoglobules and the chloroplast envelope (Lichtenthaler, 2007) which are not involved in photosynthetic electron transport.

\subsection{PQ-C determination by UPLC-MS/MS}

Hydroxyplastoquinone (PQ-C) was analyzed using an UPLC-MS/MS system (UPLC ultimate RS 3000; Thermo Fisher with QTOF 5600; AB Sciex) connected to a Kinetex C18 $2.1 \times 150 \mathrm{~mm}$ column (Phenomenex). The APCl source was operated in negative mode. A binary solvent system was used, in which mobile phase A consisted of acetonitrile:water (60:40, v/v) and $10 \mathrm{~mm}$ ammonium formate and mobile phase B consisted of isopropanol:acetonitrile (90:10, v/v), $10 \mathrm{~mm}$ ammonium formate, and $0.1 \%$ formic acid $(\mathrm{v} / \mathrm{v})$. The gradient started with $27 \%$ of solution $B$ and was increase to $97 \%$ of solution $B$ within $20 \mathrm{~min}$ at a speed of $0.3 \mathrm{ml} \mathrm{min}^{-1}$, and then maintained at $97 \%$ for $5 \mathrm{~min}$. Solution B was then decreased to a $27 \%$ enrichment during $7 \mathrm{~min}$ for column re-equilibration. Column temperature was set at $45^{\circ} \mathrm{C}$. Oxidized and reduced PQ-9 and PQ-C identification was achieved based on the comparison of retention times and accurate masses with standards. Reduced PQ-9 and PQ-C were obtained by reducing the oxidized PQ-9 and PQ-C standards with sodium borohydride. In samples, PQ-C and PQ-9 contents were measured in selected reaction monitoring (SRM) mode using the following transition: $\mathrm{m} / \mathrm{z} 764.6->\mathrm{m} / \mathrm{z} 202.095+/-0.025(\mathrm{CE}=-70 \mathrm{eV})$ and $\mathrm{m} / \mathrm{z} 748.6->\mathrm{m} / \mathrm{z} 149.058+/-0.025$ (CE = -70 eV) respectively. Examples of extracted-ion chromatograms (XIC) for PQ-C are shown in Supplementary Fig. S2.

\subsection{Chloroplast fractionation}

Chloroplasts, isolated from leaves of 6-week-old plants, were hypotonically ruptured and subplastidial compartments were isolated by centrifugation using sucrose-density gradient following the protocole described in (Besagni, Eugeni Piller, \& Bréhélin, 2011), except that plants were not pre-adapted to darkness. 32 fractions of $1 \mathrm{ml}$ were collected from the centrifuged tubes (from top to bottom), and $400 \mu$ l of each fraction were diluted to obtain a final sucrose concentration of $5 \%$. The diluted fractions were then grinded in mortar glass tube with $5 \mathrm{ml}$ of cold ethyl acetate and sonicated (Digital Sonifer, Brandon, USA) for 1 min with a 50\% 'duty cycle' at $40 \%$ of power in an ice bath. The extracts were centrifuged for $13 \mathrm{~min}$ at $9600 \mathrm{~g}$ at $4^{\circ} \mathrm{C} .500 \mu$ l of supernatant was evaporated in a stream of nitrogen and dissolved in $300 \mu \mathrm{l}$ of methanol/hexane $(17 / 1, \mathrm{v} / \mathrm{v}) .100 \mu$ l were immediately analyzed by HPLC, as described above.

\subsection{Chlorophyll fluorescence}

The maximal quantum yield of PSII photochemistry was measured on attached leaves with a PAM2000 fluorometer (Walz) as $\mathrm{Fv} / \mathrm{Fm}=(\mathrm{Fm}-\mathrm{Fo}) / \mathrm{Fm}$ where $\mathrm{Fm}$ is the maximal fluorescence level obtained 
with a 800-ms pulse of intense white light and Fo is the initial level obtained after a 2-s pulse of far-red light. Measurements were done in the dark in leaves dark-adapted for 30 min.

Chlorophyll fluorescence induction curves were measured with a JTS-10 spectrophotometer/ fluorimeter (BloLogic, France), and the number of PSII 2-electron acceptors was estimated as described in Joliot \& Joliot (2002) as the area over the fluorescence rise measured in dark-adapted leaves divided by the area over the curve for leaves infiltrated with DCMU (one electron transferred to $Q_{A}$ in the light). Chlorophyll fluorescence imaging was done with a laboratory-built instrument described in Johnson et al. (2009).

\subsection{Lipid peroxidation}

Lipids were extracted from approximately $0.5 \mathrm{~g}$ of leaves frozen in liquid nitrogen. The leaves were grinded in an equivolume methanol/chloroform solution containing 5mM Triphenyl Phosphine, $1 \mathrm{mM}$ 2,6-tert-butyl-p-cresol (BHT) (5 ml g ${ }^{-1}$ fresh weight) and citric acid ( $2.5 \mathrm{ml} \mathrm{g}^{-1}$ fresh weight), using an Ultraturax blender. Internal standard 15-HEDE was added to a final concentration $100 \mathrm{nmol} \mathrm{g}^{-1}$ fresh weight, and mixed properly. After centrifugation at $700 \mathrm{rpm}$ and $4^{\circ} \mathrm{C}$ for $5 \mathrm{~min}$, the lower organic phase was carefully taken out with the help of a glass syringe into a $15 \mathrm{ml}$ glass tube. The syringe was rinsed with approximately $2.5 \mathrm{ml}$ chloroform and transferred back into the tube. The process was repeated and the lower layer was again collected and pooled to the first collection. The solvent was evaporated under $\mathrm{N}_{2}$ gas at $40^{\circ} \mathrm{C}$. The residues were recovered in $1.25 \mathrm{ml}$ absolute ethanol and $1.25 \mathrm{ml}$ of $3.5 \mathrm{~N}$ $\mathrm{NaOH}$ and hydrolyzed at $80^{\circ} \mathrm{C}$ for $30 \mathrm{~min}$. The ethanol was evaporated under $\mathrm{N}_{2}$-gas at $40{ }^{\circ} \mathrm{C}$ for $\sim 10$ min. After cooling to room temperature, $\mathrm{pH}$ was adjusted to $4-5$ with $2.1 \mathrm{ml}$ citric acid. Hydroxy fatty acids were extracted with hexane/ether $50 / 50(\mathrm{v} / \mathrm{v})$. The organic phase was analyzed by straight phase HPLC-UV, as previously described (Montillet et al., 2004). The hydroxyoctadecatrienoic acid (HOTE) isomers (9-, 12-, 13- and 16-HOTE derived from the oxidation of the main fatty acid in Arabidopsis leaves, linolenic acid) were quantified based on the 15-HEDE internal standard.

Lipid peroxidation was also visualized in whole plants by autoluminescence imaging. Stressed plants were dark adapted for $2 \mathrm{~h}$, and the luminescence emitted from the spontaneous decomposition of lipid peroxides was captured by a highly sensitive liquid $\mathrm{N}_{2}$-cooled charge-coupled device (CCD) camera, as previously described (Birtic et al., 2011). The images were treated using Image J software (NIH, USA)

\subsection{SOSG-EP fluorescence}

Production of ${ }^{1} \mathrm{O}_{2}$ was measured in attached leaves from the fluorescence of the ${ }^{1} \mathrm{O}_{2}$-specific SOSG (Singlet Oxygen Sensor Green) fluorescent probe (Invitrogen), as described previously (Ramel et al., 
2012). With the help of a 1-ml syringe (without the needle), $100 \mu \mathrm{M}$ SOSG was pressure-infiltrated into the leaves through the lower surface. Plants with SOSG-infiltrated leaves were exposed to a PFD of $1500 \mu \mathrm{mol}$ photons $\mathrm{m}^{-2} \mathrm{~s}^{-1}$ at $7^{\circ} \mathrm{C}$ for $20 \mathrm{~min}$. As a control treatment, plants with SOSG-infiltrated leaves were placed in the dark at room temperature for $20 \mathrm{~min}$. SOSG-EP fluorescence was then measured from leaf discs punched from the SOSG-infiltrated leaves using a fiberoptics-equipped Perkin-Elmer spectrofluorometer (LS 50B) with a $475 \mathrm{~nm}$ excitation light. SOSG-EP fuorescence at $524 \mathrm{~nm}$ (F524) was normalized to chlorophyll fluorescence at $680 \mathrm{~nm}$ (F680) for each leaf disc. SOSG-EP fluorescence was calculated as: ([F524 in the light - F524 in the dark]/F680) x 10.

\subsection{EPR spectroscopy}

For the preparation of thylakoid membranes, $7 \mathrm{~g}$ of leaves (fresh weight) were grinded for $2 \mathrm{~s}$ in $50 \mathrm{ml}$ of extraction buffer ( $330 \mathrm{mM}$ sorbitol, $50 \mathrm{mM}$ Tricine, $2 \mathrm{mM}$ EDTA(Na2), $1 \mathrm{mM} \mathrm{MgCl}$, 2 mM Ascorbate, $\mathrm{pH}$ 7.7) with $5 \mathrm{mM}$ dithiothreitol (DTT) in a Warring blendor at low speed. The liquid phase was removed and set aside, and $50 \mathrm{ml}$ of extraction buffer was added for a second extraction. The extracts were filtered onto 4 Miracloth layers, and the filtrate was centrifuged for $4 \mathrm{~min}$ at $1500 \mathrm{~g}$ at $4^{\circ} \mathrm{C}$. The pellet was washed twice with the extraction buffer and centrifuged for $4 \mathrm{~min}$ at $1500 \mathrm{~g}$ at $4^{\circ} \mathrm{C}$. The washed pellet was resuspended in $21 \mathrm{ml}$ of lysis buffer $\mathrm{pH} 7.8$ (10 mM Tricine, $10 \mathrm{mM} \mathrm{NaCl}, 10 \mathrm{mM}$ $\mathrm{MgCl}_{2}$ ) with $1 \mathrm{mM}$ PMSF (phenyl methylsulfonyl) with occasional stirring for $15 \mathrm{~min}$. The sample was centrifuged at $48400 \mathrm{~g}$ for $15 \mathrm{~min}$. The pellet was resuspended in $1.75 \mathrm{ml}$ of storage buffer $(100 \mathrm{mM}$ Tricine, $10 \mathrm{mM} \mathrm{NaCl}, 10 \mathrm{mM} \mathrm{MgCl}$, $400 \mathrm{mM}$ sucrose, $\mathrm{pH} 7.8$ ) and stored at $-80^{\circ} \mathrm{C}$ before analyses.

Singlet oxygen formation was monitored by electron paramagnetic resonance (EPR) spectroscopy using the spin probe 2,2,6,6-tetramethyl-4-piperidone (TEMPD) purified by vacuum distillation. The oxidation of TEMPD by ${ }^{1} \mathrm{O}_{2}$ forms 2,2,6,6-tetramethyl-4-piperidone-1-oxyl (TEMPONE) detectable by EPR spectroscopy. Thylakoid membranes $\left(25 \mu \mathrm{g} \mathrm{Chl} \mathrm{ml} \mathrm{m}^{-1}\right)$ were illuminated in the presence of $50 \mathrm{mM}$ TEMPD and $40 \mathrm{mM}$ MES-NaOH buffer $(\mathrm{pH}=6.5)$ for $30 \mathrm{~min}$ under $1000 \mu \mathrm{mol}$ photons $\mathrm{m}^{-2} \mathrm{~s}^{-1}$. After illumination, thylakoid membranes were centrifuged at $1000 \times \mathrm{g}$ for $2 \mathrm{~min}$ to separate sample from spin probe. The EPR spectra were recorded using an EPR spectrometer Mini Scope MS400 (Magnettech GmbH, Berlin, Germany). EPR measurement conditions were as follows: microwave power, $10 \mathrm{~mW}$; modulation amplitude, $1 \mathrm{G}$; modulation frequency, $100 \mathrm{G}$; scan rate, 1.62 $\mathrm{G} \mathrm{s}^{-1}$.

\section{RESULTS}

3.1 Overexpression of the SPS1 gene in the vte1 mutant leads to a marked accumulation of PQ-9, mainly in the plastoglobules and the chloroplastic envelopes 
Ksas et al. (2015) previously showed that overexpressing the SPS1 gene in Arabidopsis brings about a noticeable increase in the leaf content in both PQ-9 and PC-8. This was confirmed here with one of the SPS10ex lines previously described, with a 50\%-increase in the total PQ-9 content and a 3-fold increase in PC-8 compared to WT (Fig. 1A). Please note that throughout the text the term PQ-9 is used as a generic term for total PQ-9, i.e. oxidized PQ-9 + reduced $\mathrm{PQH}_{2}-9$ (plastoquinol). In all genotypes, $\mathrm{PQ}-9$ was highly reduced, with $\mathrm{PQH}_{2}-9$ (plastoquinol) representing around $75 \%$ of the total pool (Supplemental Fig. S3). The tocopherol cyclase mutant vte1 is devoid of both tocopherols and PC-8 (Zbierzak et al., 2009). The loss of those two lipid-soluble antioxidants in vte1 leaves was accompanied by a significant decrease in the total PQ-9 levels, as previously reported (Ksas et al, 2015). When the vte1 mutant was crossed with the SPS1-overexpressing line (line \#12 in Ksas et al., 2015) to generate a double mutant vte1 SPS10ex, the total PQ-9 content of the leaves was strongly increased, reaching values above the WT levels. Compared to vte1, vte1 SPS1oex contained approximately 3 times more PQ-9. The changes in tocochromanols observed in vte1, SPS1oex and vte1 SPS1oex had no significant effect on the growth phenotype of the plants in low light (Fig. 1B) and did not affect significantly the efficiency of photosynthetic electron transport in young and mature leaves as measured by the chlorophyll fluorescence parameter $\Phi_{\text {PSII }}$ (Fig. 1C). Compared to the SPS1 overexpressors, a decrease in $\Phi_{\text {PSII }}$ was nevertheless observed in the older leaves of vte1 and, to a lesser extent, of WT. Thus, high PQ-9 levels appear to be helpful to maintain the efficiency of PSII-mediated electron flow in old, presenescing leaves.

To determine the localization of the extra PQ-9 that accumulated in SPS1-overexpressing plants, chloroplast membranes were fractionated by ultracentrifugation on sucrose gradient (Besagni et al., 2011). As expected (Besagni et al., 2011; Vidi et al., 2006), 3 pigmented fractions were obtained (Fig. 2A): plastoglobules (PG) were found floating at the top of the tubes and forming a whitish layer, while an intermediate yellowish zone corresponding to the outer and inner chloroplast envelope membranes (ENV) separated from the green zone at the bottom containing chlorophyll-binding thylakoid membranes. 32 fractions were collected from top (fraction 1) to bottom (fraction 32) and analyzed for their PQ-9 content (Fig. 2B, Supplemental Fig. S4). PQ-9 was found in all fractions, with an almost equivalent partitioning between the thylakoids and the two other fractions (envelopes+plastoglobules) in WT leaves (Supplemental Fig. S4). We checked the abundance of the plastoglobule marker protein Plastoglobulin 35 (PGL35) in the different fractions by Western blotting. Compared to the plastoglobule fraction, the abundance of PGL35 in the envelope fraction was very low (Supplemental Fig. S5). Consequently, the almost similar amounts of PQ-9 found in the plastoglobules and the envelopes (Supplemental Fig. S4) are not attributable to a contamination of the envelopes by plastoglobules. Surprisingly, fractions $24-28$ contain very little PQ-9 although they are green. It is possible that those fractions contain photosynthetic pigmented complexes released from 
the thylakoids during preparation. In vte1, the loss of PQ-9 concerned exclusively the PG and envelope

271 fractions (fractions 1-4 and 5-14, respectively), with the levels in the thylakoids (fractions 26-32) being virtually unchanged relative to WT. Similarly, the accumulation of total PQ-9 in SPS1oex and vte1 SPS1oex leaves impacted mainly the PG and envelope fractions. Thus, thylakoid membranes appear to accommodate a relatively constant amount of PQ-9 (presumably ensuring optimum electron transport) while the PG and envelope fractions function as PQ-9 storage sites that can absorb large variations in total PQ-9 in the chloroplasts. This was confirmed by the estimation of the photochemically active and non-active pools of PQ-9 using a previously described procedure based on HPLC analysis of reduced and oxidized PQ-9 in the dark and upon intense illumination (Ksas et al., 2015). For each genotype, we found that the pool sizes of the active plus non-active pools (Fig. 2C) were matching, within experimental accuracy, the total pool size (Fig. $1 \mathrm{~A}$; in $\mathrm{nmol} \mathrm{cm}^{-2}, 0.91 \pm 0.07$ in WT, $1.43 \pm 0.15$ in SPS1oex, $0.60 \pm 0.04$ in vte 1 and $1.73 \pm 0.08$ in vte 1 SPS1oex). Figure $2 C$ shows that the photoactive pool (reflecting the thylakoid PQ-9 pool involved in photosynthetic electron transport) showed relatively little variations in the different genotypes while the non-photoactive pool (reflecting the pool, located outside the thylakoid membranes, not involved in electron transport) exhibited strong variations, with SPS1oex and vte1 SPS1oex having by far the largest pools of extra-thylakoid PQ9 (Fig. 2C), in agreement with the analyses of Fig. 2B and Supplemental Fig. S4.

It is also possible to estimate the size of the functional pool of PSII electron acceptors by in vivo chlorophyll fluorescence measurements (Malkin \& Kok, 1966; Forbush \& Kok, 1968). The chlorophyll fluorescence induction curves of Fig. 2D showed little differences between the different genotypes, confirming that the functional pool in the thylakoids was not much affected by the total PQ-9 levels. From the fluorescence induction curves of Fig. 2D and the corresponding curves obtained in the presence of DCMU, we estimated that the number of PSII two-electron acceptors is in the range 13-16 molecules (insert of Fig. 2D), which is consistent with previous estimations in various photosynthetic organisms (Forbush \& Kok, 1968; Kolber \& Falkowski, 1993).

\subsection{Accumulation of total PQ-9 in the vte1 SPS1oex mutant compensated for the lack of tocopherols} and PC-8 and increased tolerance to photooxidative stress

Plants grown in low light $\left(150 \mu \mathrm{mol}\right.$ photons $\left.\mathrm{m}^{-2} \mathrm{~s}^{-1}\right)$ were transferred to photooxidative stress conditions $\left(1500 \mu \mathrm{mol}\right.$ photons $\left.\mathrm{m}^{-2} \mathrm{~s}^{-1}, 7^{\circ} \mathrm{C}\right)$. After $2 \mathrm{~d}$, this treatment led to leaf bleaching (Fig. $\left.3 \mathrm{~A}\right)$, low Fv/Fm ratio indicative of PSII photoinhibition (Fig. 3D) and enhanced lipid peroxidation (Fig. 3B and C) in WT leaves. These symptoms of photodamage were markedly attenuated in SPS1oex plants, as previously shown (Ksas et al., 2015). In striking contrast, vte1 exhibited a very high sensitivity to photooxidative stress, showing dramatic effects on leaves (severe bleaching and loss of turgescence), intense lipid peroxidation and drastic inhibition of PSII activity relative to WT. This confirms the 
photoprotective role of tocopherol and/or PC-8 which are absent in vte1 (Havaux, Eymery, Porfirova, Rey \& Dörmann, 2005). Interestingly, overexpression of SPS1 suppressed the photobleaching phenotype of vte1. Furthermore, vte1 SPS10ex was more tolerant to lipid peroxidation than vte1. The data of Fig. 3 thus demonstrate that total PQ-9 can compensate for the lack of tocopherol and PC-8 in vte1 leaves, leading to a markedly increased tolerance to photooxidative stress. This effect was confirmed in another SPS1-overexpressing vte1 mutant and under longer stress exposure (4 d) (Supplemental Figure S6).

\subsection{Plastoquinone as a singlet oxygen scavenger in planta}

${ }^{1} \mathrm{O}_{2}$ production by illuminated leaves was monitored by measuring the intensity of SOSG-endoperoxide (EP) fluorescence at $525 \mathrm{~nm}$ (Flors et al., 2006; Kim, Fujitsuka, \& Majima, 2013). As shown in Fig. 4A, the photosensitivity of vte1 leaves correlated with an increased production of ${ }^{1} \mathrm{O}_{2}$ compared to WT, in line with the known function of tocopherols as ${ }^{1} \mathrm{O}_{2}$ quenchers (Fahrenholtz, Doleiden, Trozzolo, \& Lamola, 1974; Di Mascio, Devasagayam, Kaiser, \& Sies, 1990; Krieger-Liszkay, Fufezan, \& Trebst, 2008; Choe, 2107). It was decreased in vte1 SPS1oex, showing that replacement of tocopherols by total PQ9 restores the ${ }^{1} \mathrm{O}_{2}$ quenching capacities. This finding was confirmed by the analysis of ${ }^{1} \mathrm{O}_{2}$ production by illuminated thylakoid membranes using EPR spectroscopy and the ${ }^{1} \mathrm{O}_{2}$-specific spin probe TEMPONE (Fig. 4C-D). The production rate of ${ }^{1} \mathrm{O}_{2}$ was the highest in vte1 thylakoid membranes, whereas accumulation of PQ-9 by SPS1 overexpression in both WT and vte1 backgrounds resulted in the lowest ${ }^{1} \mathrm{O}_{2}$ production levels in the light, emphasizing the role of total PQ-9 in the quenching of ${ }^{1} \mathrm{O}_{2}$ in vivo.

Oxidation of PQ-9 by ${ }^{1} \mathrm{O}_{2}$ has been shown to produce some specific derivatives, such as the hydroxy derivative PQ-C (Szymanska et al., 2014). The latter compound was shown to be a stable product of ${ }^{1} \mathrm{O}_{2}$ action in plants, particularly when ${ }^{1} \mathrm{O}_{2}$ production is elevated during short-term exposure to high light stress. Quantification of PQ-C in control Arabidopsis leaves showed the presence of this compound in all genotypes (Fig. 4B). Similarly to PQ-9, PQ-C was predominantly in the reduced state $\left(\mathrm{PQH}_{2}-\mathrm{OH}\right)$ (Supplemental Fig. S3). $\mathrm{PQ}-\mathrm{C}$ accumulation is consistent with the attribution of a ${ }^{1} \mathrm{O}_{2}$ scavenging function to PQ-9: the amount of this PQ-9 hydroxy derivative, formed by ${ }^{1} \mathrm{O}_{2}$, is the highest in vte1 SPS10ex where total PQ-9 is the most abundant and is the major lipid-soluble antioxidant in the absence of tocopherol and PC-8.

\subsection{High light stress preferentially consumes the thylakoidal PQ-9 pools} Arabidopsis plants were exposed for $2.5 \mathrm{~h}$ to high PFD (1500 $\mu \mathrm{mol}$ photons $\left.\mathrm{m}^{-2} \mathrm{~s}^{-1}\right)$ and low temperature $\left(7^{\circ} \mathrm{C}\right)$. The total PQ-9 concentration was decreased by around $30 \%$ in WT leaves after this treatment (Fig. 5A). Fig. 5B shows that the PQ-9 losses were particularly marked for the thylakoidlocated photochemically active PQ-9 pool (-60\%) while the extra-thylakoid, photochemically non- 
active pool was not significantly affected. Since the photosystems in the thylakoids are the main source of ROS in the light, a preferential oxidation of this photochemically active pool is expected. This is actually confirmed by the analysis of ${ }^{1} \mathrm{O}_{2}$-specific $\mathrm{PQ}-\mathrm{C}$ compound in the different membrane fractions of the chloroplasts: PQ-C was predominantly found in the thylakoids, with the concentration in the plastoglobules and the envelopes being very low (Fig. 5C). In vte1, the photoinduced decrease in total PQ-9 content was very marked (>50\%) (Fig. 5A), and the photochemically active pool was almost completely depleted after the high light treatment (Fig. 5B). However, contrary to WT, the size of the extra-thylakoid, photochemically non-active PQ-9 pool was also decreased in the mutant, although to a lesser extent (-40\%) than the active pool (Fig. 5B). Fig. 5C shows that, similarly to WT, PQ-C in vte1 chloroplasts was present mainly in the thylakoids, confirming that PQ-9 oxidation occurred at this level. As shown at the leaf level (Fig. 3), the PQ-C concentration was noticeably higher in vte1 thylakoids compared to WT thylakoids, indicating enhanced oxidation of PQ-9 in this mutant. In vte1 SPS1oex leaves which rely on high levels of PQ-9 for their phototolerance, a considerable loss of total PQ-9 was observed during high light stress (Fig. 5A, 5B), with a very strong accumulation of PQ-C in the thylakoids (Fig. 5C). Thus, our data suggest that, under conditions of massive oxidative degradation of PQ-9 in the thylakoids of vte1 and vte1 SPS1oex chloroplasts, there is a transfer of PQ-9 molecules from the plastoglobules and/or the envelope to the thylakoid membranes in order to compensate for PQ-9 photooxidation, hence lowering the PQ-9 levels in the storage sites outside the thylakoid membranes. Owing to their high accumulation levels of total PQ-9, vte1 SPS1oex leaves were able to maintain the total PQ-9 content close to the WT levels after the high light treatment (Fig. 5A), and this was associated with a high resistance to photooxidative stress (Fig. 3).

\section{DISCUSSION}

The essential role of PQ-9 in photosynthesis as an electron carrier has long been recognized (Amesz, 1973; Crane, 2010). However, it is clear from this study that PQ-9 also fulfills an important photoprotective function that is not directly related to photosynthetic electron transport. Actually, only a fraction of the total PQ-9 pool is located in the thylakoid membranes where it can participate in linear and cyclic electron fluxes. We estimated that, in WT Arabidopsis leaves under normal growth conditions, the photochemically active PQ-9 corresponds to around 15 molecules per PSII and $50 \%$ of the total PQ-9 content. The latter value is higher than previous reports (ca. 30\%, Kruk \& Karpinski, 2006; Szymanska \& Kruk 2010), possibly due to different growth conditions. The size of the total thylakoid-associated PQ-9 pool was found to be stable in control conditions, showing relatively little variation among the different genotypes studied here or in response to changes in the total PQ-9 content of the leaves. This stability contrasts with the non-photochemically active pool, the size of which exhibited large variations. As confirmed in this study, the latter PQ-9 pool partitions between 
the plastoglobules (Zbierzak et al., 2009) and the envelope membranes (Soll et al., 1985). The size of the extra-thylakoid PQ-9 pool drastically decreased in the photosensitive vte1 mutant, whereas it was selectively increased when PQ-9 biosynthesis was stimulated. The highest PQ-9 levels were found in vte1 SPS10ex leaves in which approximately two-thirds of the total PQ-9 pool was not in the thylakoids. In contrast, only $40 \%$ of the total pool was outside the thylakoid membrane in the vte1 single mutant that contains the lowest levels of total PQ-9. Somehow, the PQ-9 reservoir stored in the plastoglobules and the chloroplast envelopes functions as a buffer that can manage large variations in the total PQ-9 levels in the leaves.

The multi-location storage of PQ-9 suggests a multi-functionality for this compound. Previously, PQ-9 was reported to be involved in biosynthesis pathways, as a component of phytoene desaturase in the carotenoid biosynthesis pathway (Norris et al., 1995) and of NDC1, a type-II NAD(P)H quinone oxidoreductase involved in vitamin K1 synthesis (Eugeni Piller et al., 2011). This work shows that it is also a ROS scavenger protecting the chloroplasts against photodamage by a mechanism that could possibly be similar to the antioxidant activity of tocopherol. Indeed, overexpression of SPS1 in the tocopherol-deficient vte1 mutant concomitantly enhanced leaf PQ-9 levels and decreased plant photosensitivity, indicating that PQ-9 can replace tocopherols in photoprotection. Tocopherol has been shown to act as a ${ }^{1} \mathrm{O}_{2}$ quencher (Fahrenholtz et al., 1974; Di Mascio et al., 1990; Krieger-Liszkay et al., 2008; Choe, 2017) and a terminator of lipid peroxidation (Burton \& Ingold, 1986; Ham \& Liebler, 1995). PQ-9 can also do both. Indeed, Nowicka et al. (2013) showed that reduced or oxidized PQ-9 incorporated into liposomes precludes lipid oxidation, and the present work demonstrates that high total PQ-9 levels lowered ${ }^{1} \mathrm{O}_{2}$ accumulation levels. Moreover, high light stress leads to a loss of PQ-9 and a concomitant formation of PQ-C indicating ${ }^{1} \mathrm{O}_{2}$ oxidation of PQ-9. As expected, the accumulation of PQ-C was the highest in vte1 SPS1oex leaves which rely the most on total PQ-9 for their resistance to photooxidative stress. PQ-C was found almost exclusively in the thylakoids where ROS are produced in the light, either by excitation energy transfer $\left({ }^{1} \mathrm{O}_{2}\right)$ or by electron transfers (superoxide anion radical, hydrogen peroxide or hydroxyl radical) (Asada, 2006; Krieger-Liszkay et al. , 2008; Li, Wakao, Fischer \& Niyogi, 2009; Pospisil, 2014).

PQ-9, especially when it is in the reduced state, possesses a number of features that are likely to make this compound an excellent antioxidant. Firstly, it is present in high amounts as a diffusible molecule in thylakoids. As shown in Fig. 1, the concentration of total PQ-9 in Arabidopsis leaves is higher than tocopherol + plastochromanol levels. Leaf enrichment in total PQ-9 relative to tocopherols can be even amplified during long-term acclimation of plants to high light intensities (Ksas et al., 2015; Lichtenthaler, 2007). Second, the PQ-9 side chain bears several double bonds contrary to tocopherols. Double bonds are known to be preferential targets of ${ }^{1} \mathrm{O}_{2}$ for oxidation (Triantaphylidès \& Havaux, 2009), and therefore the unsaturated side-chain of PQ-9 could provide a possibility for PQ-9 to quench 
${ }^{1} \mathrm{O}_{2}$ by a chemical mechanism. Nevertheless, the antioxidant activity is mainly beard by the phenolic group of reduced PQ-9 (Kim \& Min, 2008), so that reduced PQ-9 is a better ${ }^{1} \mathrm{O}_{2}$ quencher than oxidized PQ-9. Third, in thylakoid membranes, photosynthetic electron transport relies on the interaction between PQ-9 and the PSII reaction centers which are the main ${ }^{1} \mathrm{O}_{2}$ generators during photosynthesis (Krieger-Liszkay et al., 2008). It was initially hypothesized that, in the thylakoid membranes, PQ-9 is located in the fluid bilayer-midplane region allowing rapid lateral movements between cytochrome b6/f and PSII complexes (Millner \& Barber, 1984). However, more recent data favor a close compartmentization of PSII, PQ-9 and cytochrome b6/f complex in membrane microdomains (Lavergne \& Joliot, 1991; Johnson, Vasilev, Olsen, \& Hunter, 2014). Moreover, PQ-9 diffusion towards/from PSII was recently proposed to occur via several entries/exits in the PSII reaction center with an exchange cavity where PQ-9 can diffuse around (Van Eerden, Melo, Frederix, Periole, \& Marrink, 2017). These characteristics of the interactions between PSII and PQ-9 are likely to augment the opportunities for PQ-9 to scavenge ${ }^{1} \mathrm{O}_{2}$ produced in the reaction centers. Consequently, PQ-9 could constitute a first line of defense against photosynthesis-produced ROS, supplementing the action of the $\beta$-carotene molecules bound to the PSII centers.

Chemical quenching involves oxidation of the quencher and therefore total PQ-9 is expected to be consumed during its antioxidant activity, as indeed observed in plants exposed to photooxidative stress conditions (Ksas et al., 2015; Fig. 5). In the vte1 mutant, the total PQ-9 levels are constitutively lower than in WT, and this can be attributed to the chronic oxidation of PQ-9 in the absence of tocopherol. The increased levels of PQ-C in the vte1 mutants compared to WT corroborate this interpretation. This phenomenon concerns mainly the extra-thylakoid PQ-9 pool, with the total thylakoid-located PQ-9 levels being virtually unchanged relative to WT levels under control conditions. Since ROS are produced in the light in the thylakoids, a dynamic exchange of PQ-9 molecules must exist between the thylakoids and their storage sites in the plastoglobules and the envelopes to replace photooxidized PQ-9 molecules in the thylakoid membranes with new PQ-9 molecules and to maintain a constant pool in the thylakoid membranes. Accordingly, plastoglobules have been shown to be physically coupled to thylakoids in a way allowing bidirectional channeling of lipid metabolites (Austin, Frost, Vidi, Kessler, \& Staehelin, 2006, Bréhélin \& Kessler, 2008). However, experimental data measuring the nature and the rate of this metabolite exchange are not available. When Arabidopsis plants were suddenly exposed to high light stress, total PQ-9 levels drastically decreased in the thylakoids, as expected when excess light energy leads to high ${ }^{1} \mathrm{O}_{2}$ production levels by the photosystems. Moreover, $\mathrm{PQ}-\mathrm{C}$ was detected predominantly in the thylakoid fractions, indicating that $442{ }^{1} \mathrm{O}_{2}$ oxidation of PQ-9 occurs at this level. This is in agreement with a previous study by Szymanska and Kruk (2010) who showed a selective decrease in the photochemically active PQ-9 in Arabidopsis leaves exposed to high light stress (Szymanska \& Kruk, 2010). However, we also observed a significant 
reduction of the total PQ-9 concentration, though less pronounced, in the photochemically non-active pool when the photostress was severe and PQ-9 degradation was very pronounced such as in the vte1 background. This effect of high light stress is consistent with a transfer of PQ-9 molecules from the plastoglobules and/or envelopes to the thylakoids to compensate the oxidative modification of PQ-9 during its scavenging activity. In this context, it is worth mentioning that reduced PQ-9 showed a high mobility in lipid membranes compared to $\alpha$-tocopherol (Jemiola-Rzeminska, Kruk, \& Strzalka, 2003). Nevertheless, the rate of this transfer appeared to be slow compared to the rate of PQ-9 oxidation in high light since it could not maintain the pool size of photochemically active PQs to the control levels measured in low light.

Because PQ-9 somehow functions as a sacrificial antioxidant consumed during oxidative stress, it must be re-synthesized for the pool being re-filled. Accordingly, most genes of the PQ-9 biosynthesis pathway are strongly upregulated during acclimation of Arabidopsis to high light intensities (Ksas et al., 2015; Block et al., 2013). This induction of PQ-9 biosynthesis genes was accompanied by a concomitant rise in the size of the total PQ-9 pool while there was less effect on the tocopherol content (Ksas et al., 2015; Szymanska \& Kruk, 2010). The selective accumulation of PQ-9 molecules during growth in high light environments was reported in other species such as beech or fig trees (Lichtenthaler, 2007), consistently with the photoprotective role described for this compound in the present work.

\section{ACKNOWLEDGMENTS}

We would like to thank the Phytotec platform (Commissariat à l'Energie Atomique et aux Energies Alternatives, Cadarache) for growing plants under normal and stress conditions. We also thank Jerzy Kruk for providing us with PQ-9 and PC-8 standards. This work was supported by the French National Research Agency (ANR project SLOSAM, 14-CE02-0010-02). The PP laboratory was supported by the Ministry of Education, Youth and Sports of the Czech Republic grant LO1204 (Sustainable development of research in the Centre of the Region Haná from the National Program of Sustainability I). The UPLCMS/MS of the HelioBiotec platform was funded by the European Union, the Région Provence-AlpesCôte d'Azur, the French Ministry of Research and CEA.

\section{CONFLICT OF INTEREST}

The authors claim no conflict of interest

\section{REFERENCES}

Amesz, J. (1973) The function of plastoquinone in photosynthetic electron transport. Biochimica et Biophysica Acta, 301, 35-51. 
Asada, K. (2006) Production and scavenging of reactive oxygen species in chloroplasts and their functions. Plant Physiology, 141, 391-396.

Austin, J.R. 2nd, Frost, E., Vidi, P.A., Kessler, F., \& Staehelin, L.A. (2006) Plastoglobules are lipoprotein subcompartments of the chloroplast that are permanently coupled to thylakoid membranes and contain biosynthetic enzymes. Plant Cell, 18, 1693-1703.

Besagni, C., Eugeni Piller, L., \& Bréhélin, C. (2011) Preparation of plastoglobules from Arabidopsis plastids for proteomic analysis and other studies. Methods in Molecular Biology, 775, 223-239.

Birtic, S., Ksas, B., Genty, B., Mueller, M.J., Triantaphylides, C., \& Havaux, M. (2011) Using spontaneous photon emission to image lipid oxidation patterns in plant tissues. Plant Journal, 67, 1103-1115.

Block, A., Fristedt, R., Rogers, S., Kumar, J., Barnes, B., Barnes, J., Elowsky, C.G., Wamboldt, Y., Mackenzie, S.A., Redding, K., Merchant, S.S., \& Basset, G.J. (2013) Functional modeling identifies paralogous solanesyl-diphosphate synthases that assemble the side chain of plastoquinoe-9 in plastids. Journal of Biological Chemistry, 200, 27594-27606

Bréhélin, C., \& Kessler, F. (2008) The plastoglobule: a bag full of lipid biochemistry tricks. Photochemistry and Photobiology, 84,1388-1394.

Burton, G.W., \& Ingold, K.U. (1986) Vitamin E: Application of the Principles of Physical Organic Chemistry to the Exploration of Its Structure and Function. Accounts of Chemical Research, 19, 194201.

Choe, E. (2017) Effects and mechanism of minor compounds in oil on lipid oxidation. In Food Lipids: Chemistry, Nutrition, and Biotechnology (ed C.A. Koh), pp. 567-590. CRC Press, Boca Raton.

Cook, W.B., \& Miles, D. (1992) Nuclear mutations affecting plastoquinone accumulation in maize. Photosynthesis Research, 31, 99-111.

Crane, F.L. (2010) Discovery of plastoquinone: a personal perspective. Photosynthesis Research, 103, 195-209.

Di Mascio, P., Devasagayam, T.P., Kaiser, S., \& Sies, H. (1990) Carotenoids, tocopherols and thiols as biological singlet molecular oxygen quenchers. Biochemical Society Transactions, 18, 1054-1056.

Eugeni Piller, L., Besagni, C., Ksas, B., Rumeau, D., Bréhélin, C., Glauser, G., Kessler, F., \& Havaux, M. (2011) Chloroplast lipid droplet type II NAD(P)H quinone oxidoreductase is essential for prenylquinone metabolism and vitamin K1 accumulation. Proceedings National Academy of Sciences USA, 108;14354-14359.

Fahrenholtz, S.R., Doleiden, F.H., Trozzolo, A.M., \& Lamola, A.A. (1974) On the quenching of singlet oxygen by alpha-tocopherol. Photochemistry and Photobiology, 20, 505-509.

Falk, J., \& Munné-Bosch, S. (2010) Tocochromanol functions in plants: antioxidant and beyond. Journal of Experimental Botany, 61, 1549-1566. 
Flors, C., Fryer, M.J., Waring, J., Reeder, B., Bechtold, U., Mullineaux, ..., Baker, N.R. (2006) Imaging the production of singlet oxygen in vivo using a new fluorescent sensor, Singlet Oxygen Sensor Green. Journal of erimental Botany, 57, 1725-1734.

Forbush, B., \& Kok, K. (1968) Reaction between primary and secondary electron acceptors of photosystem II of photosynthesis. Biochimica et Biophysica Acta, 162, 243-253.

Gruszka, J., Pawlak, A., \& Kruk, J. (2008) Tocochromanols, plastoquinol, and other biological prenyllipids as singlet oxygen quenchers - determination of singlet oxygen rate constants and oxidation products. Free Radicals in Biology and Medecine, 45, 920-928.

Ham, A.-J. L., \& Liebler, D.C. (1995) Vitamin E Oxidation in Rat Liver Mitochondria. Biochemistry, 34, 5754-5761.

Havaux, M., Eymery, F., Porfirova, S., Rey, P., \& Dörmann, P. (2005) Vitamin E protects against photoinhibition and photooxidative stress in Arabidopsis thaliana. Plant Cel, 17, 3451-3469.

Hernandez, V.A., Eriksson, E.K., \& Edwards, K. (2015) Ubiquinone-10 alters mechanical properties and increases stability of phospholipid membranes. Biochimica et Biophysica Acta, 1848, 2233-2243.

Hundal, T., Forsmark-Andrée, P., Ernster, L., \& Andersson, B. (1995) Antioxidant activity of reduced plastoquinone in chloroplast thylakoid membranes. Archives Biochemistry Biophysics, 324, $117-$ 122.

Jemiola-Rzeminska, M., Kruk, J., and Strzalka, K. (2003) Anisotropy measurements of intrinsic fluorescence of prenyllipids reveal much higher mobility of plastoquinol than alpha-tocopherol in model membranes. Chemistry and Physics of Lipids, 123, 233-243.

Johnson, M.P., Vasilev, C., Olsen, J.D., \& Hunter, C.N. (2014) Nanodomains of cytochrome b6f and photosystem II complexes in spinach grana thylakoid membranes. Plant Cell, 26, 3051-3061.

Johnson, X., Vandystadt, G., Bujaldon, S., Wollman, F.-A., Dubois, R., Roussel, P., ..., Béal, D. (2009) A new setup for in vivo fluorescence imaging of photosynthetic activity. Photosynthesis Research, $102,85-93$.

Joliot, P., \& Joliot, A. (2002) Cyclic electron transfer in plant leaf. Proceedings National Academy of Sciences USA, 99, 10209-10214.

Kim, H.J., \& Min, D.B. (2008) Tocoperol stability and prooxidant mechanisms of oxidized tocopherols in lipids. In Food Lipids: Chemistry, Nutrition, and Biotechnology (eds C.A. Koh \& Min D.B.), pp. 435447. CRC Press, Boca Raton.

Kim, S., Fujitsuka, M., \& Majima, T. (2013) Photochemistry of singlet oxygen sensor green. Journal Physical Chemistry B, 117, 13986-13992.

Kolber, Z., \& Falkowski, P.G. (1993) Use of active fluorescence to estimate phytoplankton photosynthesis in situ. Limnology and Oceanography, 38, 1646-1665. 
Krieger-Liszkay, A., Fufezan, C., \& Trebst, A. (2008) Singlet oxygen production in photosystem II and related protection mechanism. Photosynthesis Research, 98, 551-561.

Kruk, J., \& Karpinski, S. (2006) An HPLC-based method of estimation of the total redox state of plastoquinone in chloroplasts, the size of the photochemically active plastoquinone-pool and its redox state in thylakoids of Arabidopsis. Biochimica et Biophysica Acta, 1757, 1669-1675.

Kruk, J., \& Trebst, A. (2008) Plastoquinol as a singlet oxygen scavenger in photosystem II. Biochimica et Biophysica Acta, 1777, 154-162.

Kruk, J., Szymanska, R., Cela, J., \& Munne-Bosch, S. (2014) Plastochromanol-8: Fifty years of research. Photochemistry, 108, 9-16.

Ksas, B., Becuwe, N., Chevalier, A., \& Havaux, M. (2015) Plant tolerance to excess light energy and photooxidative damage relies on plastoquinone biosynthesis. Scientific Reports, 5, 10919.

Lavergne, J., \& Joliot, P. (1991) Restricted diffusion in photosynthetic membranes. Trends in Biochemical Sciences, 16, 129-134.

Li, Z., Wakao, S., Fischer, B.B., \& Niyogi, K.K. (2009) Sensing and responding to excess light. Annual Review Plant Biology, 60, 239-260.

Lichtenthaler, H.K., \& Weinert, H. (1970) The correlation between lipoquinone accumulation and plastoglobuli formation in the chloroplasts of Ficus elastica Roxb. Zeitschrift für Naturforschung, 25b, 619-623266,

Lichtenthaler, H.K. (2007) Biosynthesis, accumulation and emission of carotenoids, alpha-tocopherol, plastoquinone, and isoprene in leaves under high photosynthetic irradiance. Photosynthesis Research, 92, 163-179.

Malkin, S., \& Kok, B. (1966) Fluorescence induction studies in isolated chloroplasts. I. Number of components involved in the reaction and quantum yields. Biochimica et Biophysica Acta, 126, 413432.

Millner, P.A., \& Barber, J. (1984) Plastoquinone as a mobile redox carrier in the photosynthetic membrane. FEBS Letters, 169, 1-6.

Montillet, J.-L., Cacas, J.-L., Garnier, L., Montane, M.H., Douki, T., Bessoule, J.J., ..., Triantaphylides, C. (2004) The upstream oxylipin profile of Arabidopsis thaliana: A tool to scan for oxidative stresses. Plant Journal, 40, 439-451.

Norris, S.R., Barrette, T.R., \& DellaPenna, D. (1995) Genetic dissection of carotenoid synthesis in arabidopsis defines plastoquinone as an essential component of phytoene desaturation. Plant Cell, 7, 2139-2149.

Nowicka, B., \& Kruk, J. (2010) Occurrence, biosynthesis and function of isoprenoid quinones. Biochimica et Biophysica Acta, 1797, 1587-1605. 
Nowicka, B., Gruszka, J., \& Kruk, J. (2013) Function of plastochromanol and other biological prenyllipids in the inhibition of lipid peroxidation- A comparative study in model systems. Biochimica et Biophysica Acta, 1828,233-240.

Pfannschmidt, T., Bräutigam, K., Wagner, R., Dietzel, L., Schröter, Y., Steiner, S., \& Nykytenko, A. (2009) Potential regulation of gene expression in photosynthetic cells by redox and energy state: approaches towards better understanding. Annals of Botany, 103, 599-607.

Porfirova, S., Bergmuller, E., Tropf, S., Lemke, R., \& Dörmann, P. (2002) Isolation of an Arabidopsis mutant lacking vitamin $\mathrm{E}$ and identification of a cyclase essential for all tocopherol biosynthesis. Proceedings National Academy Sciences USA, 99, 12495-12500.

Pospísil, P. (2014) The role of metals in production and scavenging of reactive oxygen species in photosystem II. Plant Cell Physiology, 55, 1224-1232.

Ramel, F., Birtic, S., Cuiné, S., Triantaphylidès, C., Ravanat, J.L., \& Havaux, M. (2012) Chemical quenching of singlet oxygen by carotenoids in plants. Plant Physiology, 158, 1267-1278.

Rochaix, J.D. (2014) Redox regulation of thylakoid protein kinases and photosynthetic gene expression. Antioxidants and Redox Signaling, 18, 2184-2201.

Soll, J., Schultz, G., Joyard, J., Douce, R., \& Block, M.A. (1985) Localization and synthesis of prenylquinones in isolated outer and inner envelope membranes from spinach chloroplasts. Archives of Biochemistry and Biophysics, 238, 290-299.

Szymańska, R., Nowicka, B., \& Kruk, J. (2014) Hydroxy-plastochromanol and plastoquinone-C as singlet oxygen products during photo-oxidative stress in Arabidopsis. Plant and Cell Environment, 37, 14641473.

Szymanska, R., \& Kruk, J. (2010) Plastoquinol is the Main Prenyllipid Synthesized During Acclimation to High Light Conditions in Arabidopsis and is Converted to Plastochromanol by Tocopherol Cyclase. Plant and Cell Physiology, 51, 537-546

Triantaphylides, C., \& Havaux, M. (2009) Singlet oxygen in plants: production, detioxification and signaling. Trends in Plant Science, 14, 219-228.

Van Eerden, F.J., Melo, M.N., Frederix, P.W.J.M., Periole, X., \& Marrink, S.J. (2017) Exchange pathways of plastoquinone and plastoquinol in the photosystem II complex. Nature Communications, 8,15214 .

Vidi, P.A., Kanwischer, M., Baginsky, S., Austin, J.R., Csucs, G., Dörmann, P., ..., Bréhélin, C. (2006) Tocopherol cyclase (VTE1) localization and vitamin E accumulation in chloroplast plastoglobule lipoprotein particles. Journal of Biological Chemistry, 281,11225-11234.

Yadav, D.K., Kruk, J., Sinha, R.K., \& Pospisil, P. (2010) Singlet oxygen scavenging activity of plastoquinol in photosystem II of high plants: Electron paramagnetic resonance spin-trapping study. Biochimica et Biophysica Acta, 1797, 1807-1811. 
617 Zbierzak, A.M., Kanwischer, M., Wille, C., Vidi, P.A., Giavalisco, P., Lohmann, A., .., Dörmann, P. (2009)

618 Intersection of the tocopherol and plastoquinone metabolic pathways at the plastoglobule.

619 Biochemical Journal, 425, 389-399.

620

621 


\section{Figure legends}

FIGURE 1 Accumulation of PQ-9 in the Arabidopsis vte1 SPS1oex plants. A) $\alpha$-tocopherol ( $\alpha$-Toc), PC8 and PQ-9 concentrations in leaves of WT Arabidopsis, the single mutant vte1, the SPS1 overexpressor SPS10ex and the double mutant vte1 SPS1oex (obtained by crossing vte1 with the SPS1oex line \#12 previously described in Ksas et al. (2015)). Data are mean values of 4 separate measurements $\pm S D$. B) Picture of the different genotypes at the age of 4 weeks. C) Images of the efficiency of PSII-mediated electron transport $\Phi_{\text {PSII }}$ in plants illuminated with red light of PFD $230 \mu \mathrm{mol}$ photons $\mathrm{m}^{-2} \mathrm{~s}^{-1} .{ }^{*}$ and $* * *$, different from WT at $\mathrm{P}<0.01$ and 0.001 (Student's t-test).

FIGURE 2 Localization of PQ-9 in the chloroplasts. A) Fractionation of chloroplast membranes by ultracentrifugation on sucrose gradient showing zones corresponding to the plastoglobules (PG), the envelopes and the thylakoids. The scale on the right shows the position of the different fractions collected for the PQ-9 analyses. B) Quantification of PQ-9 in 1-ml fractions from top (fraction 1) to bottom (fraction 32) for WT, vte1, SPS1oex and vte1 SPS1oex leaves. The same amounts of chlorophyll were loaded on the sucrose gradient. C) Estimation of the photochemically active and non-active pools of PQ-9 in Arabidopsis leaves. Data are mean values of 4 separate measurements \pm SD. D) Induction of chlorophyll fluorescence in intact leaves. Insert: pool sizes of PSII 2-electron acceptors estimated from the fluorescence induction curves in the presence and absence of DCMU. ${ }^{* *}$ and ${ }^{* * *}$; different from WT at $P<0.005$ and $p<0.001$ (Student's t-test).

FIGURE 3 PQ-9 accumulation increases plant tolerance to photooxidative stress. Plants were exposed for $2 \mathrm{~d}$ to high light stress $\left(1500 \mu \mathrm{mol}\right.$ photons $\left.\mathrm{m}^{-2} \mathrm{~s}^{-1}\right)$ and low temperature $\left(7^{\circ} \mathrm{C}\right)$. A) Picture of the plants after the high light treatment. B) Autoluminescence imaging of lipid peroxidation after high light treatment. The color palette shows the luminescence signal intensity from low (black) to high values (white). C) HOTE levels in leaves (in nmoles per mg fresh weight (F.W.)) before and after the high light stress. Data are mean values of 3 or 4 separate measurements \pm SD. D) Maximal PSIl photochemical efficiency, as measured by the Fv/Fm chlorophyll fluorescence ratio, before and after the high light stress. data are mean values of 8-to-12 separate measurements \pm SD. $*, * *$ and $* * *$, different from WT and $\mathrm{P}<0.01,0.005$ and 0.001 (Student's t-test).

FIGURE $4{ }^{1} \mathrm{O}_{2}$ scavenging by PQ-9. A) ${ }^{1} \mathrm{O}_{2}$ production by leaves in the light as measured by SOSG-EP fluorescence. Attached leaves infiltrated with SOSG were exposed for $20 \mathrm{~min}$ to light $(1500 \mu \mathrm{mol}$ photons $\mathrm{m}^{-2} \mathrm{~s}^{-1}$ ) at $7^{\circ} \mathrm{C}$. Data are mean values of minimum 4 or 5 measurements. B) Total PQ-C levels in leaves before and after high light stress $\left(1500 \mu \mathrm{mol}\right.$ photons $\mathrm{m}^{-2} \mathrm{~s}^{-1}$ for $2 \mathrm{~d}$ at $\left.7^{\circ} \mathrm{C}\right)$. Data are mean 
656 values (in nmoles per mg leaf fresh weight) of 3 separate measurements \pm SD. C-D) ${ }^{1} \mathrm{O}_{2}$ production by 657 thylakoids as measured by the spin probe TEMPONE and EPR spectroscopy. No signal was detected in 658 the dark. C) TEMPONE EPR traces and D) quantification of the TEMPONE EPR signal. Data are mean 659 values of 3 separate measurements \pm SD. ${ }^{*}, * *$ and ${ }^{* * *}$, different from $\mathrm{WT}$ at $\mathrm{P}<0.01, \mathrm{P}<0.005$ and $\mathrm{P}$ $<0.001$ (Student's t-test).

661

662 FIGURE 5 Dynamics of the PQ-9 pools. Effects of high light stress ( $\mathrm{HL}=2.5 \mathrm{~h}$ at $1500 \mu \mathrm{mol}$ photons $\mathrm{m}^{-}$ $6632^{2} \mathrm{~s}^{-1}$ ) on A) the PQ-9 levels in Arabidopsis leaves and B) on the repartition of PQ-9 between the 664 photochemically active pool (in the thylakoids) and the non-active pool (in the plastoglobules and the 665 envelopes). Data are mean values of mimum 3 separate measurements \pm SD. C) PQ-C concentration 666 in different chloroplast fractions, plastoglobules (PG), envelopes (ENV) and thylakoids separated by 667 ultracentrifugation on sucrose gradient (fractions 1-4, 5-10 and 30-32, respectively, see Fig. 2B). LL 668 corresponds to the growth light conditions $\left(150 \mu \mathrm{mol}\right.$ photons $\left.\mathrm{m}^{-2} \mathrm{~s}^{-1}\right)$. Data are mean values of 3 669 experiments $\pm \mathrm{SD} .{ }^{*}, * *$ and ${ }^{* * *}$, different from $\mathrm{LL}$ at $\mathrm{P}<0.01, \mathrm{P}<0.005$ and $\mathrm{P}<0.001$ (Student's t670 test). 

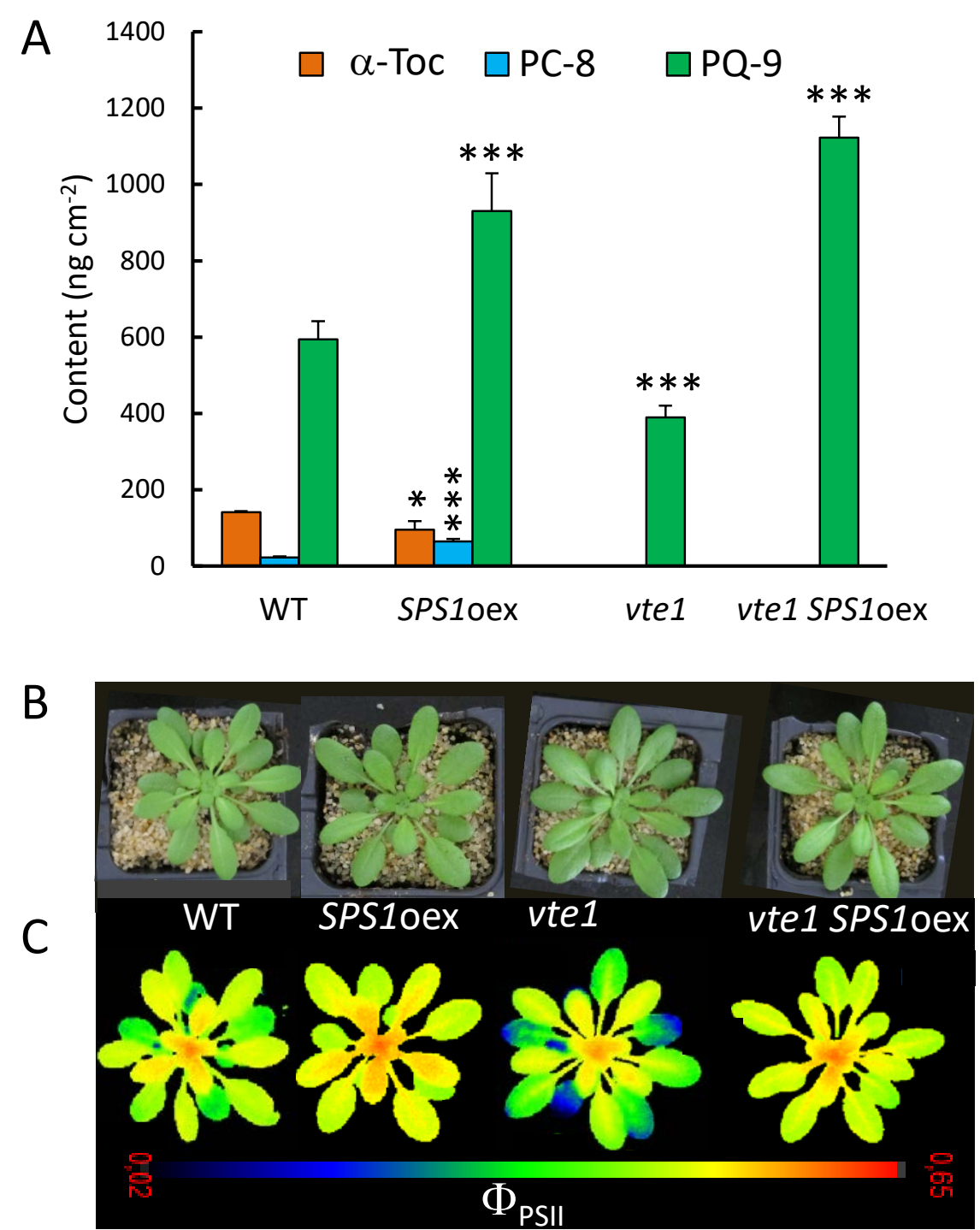

Figure 1 

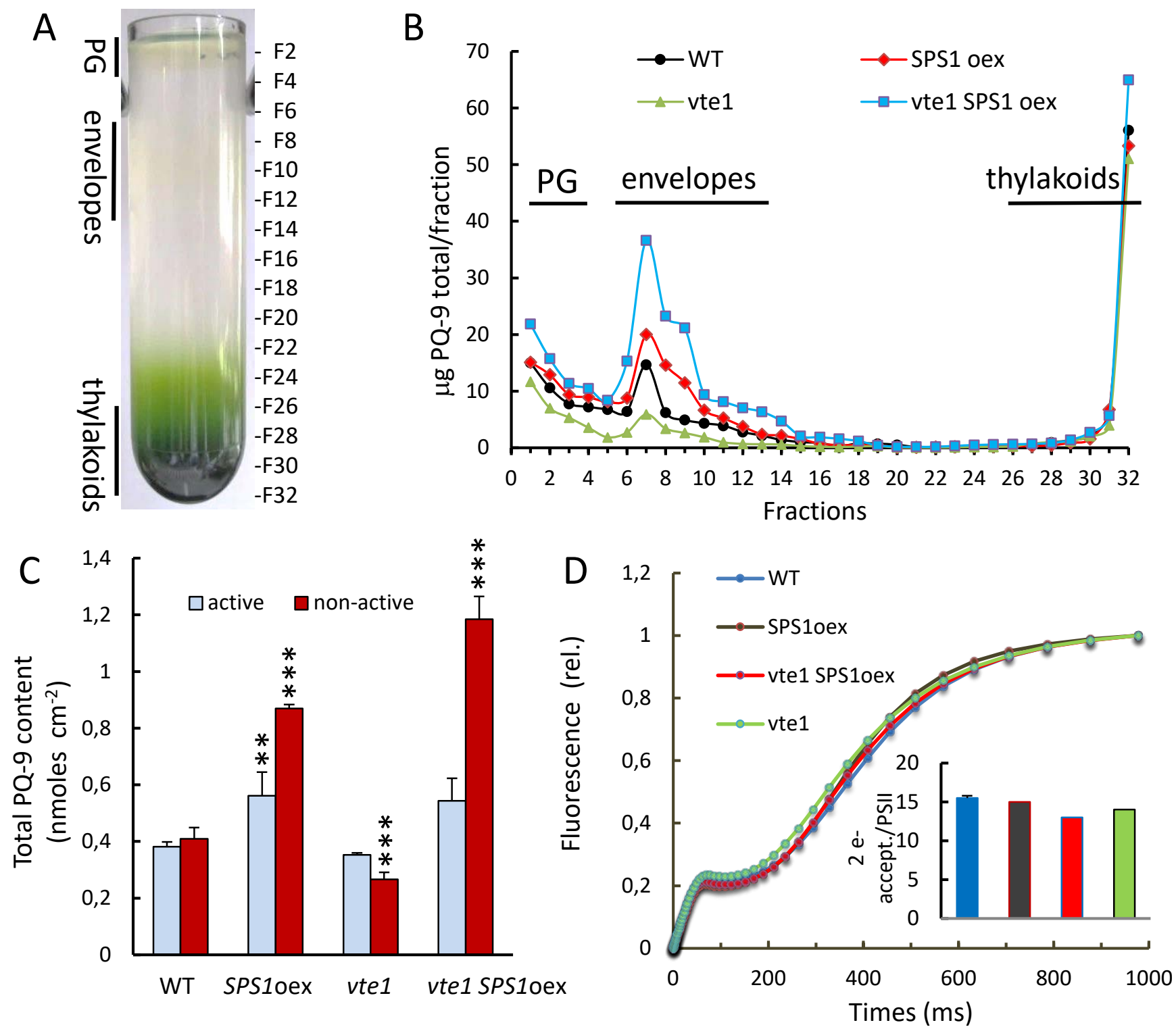

Figure 2 

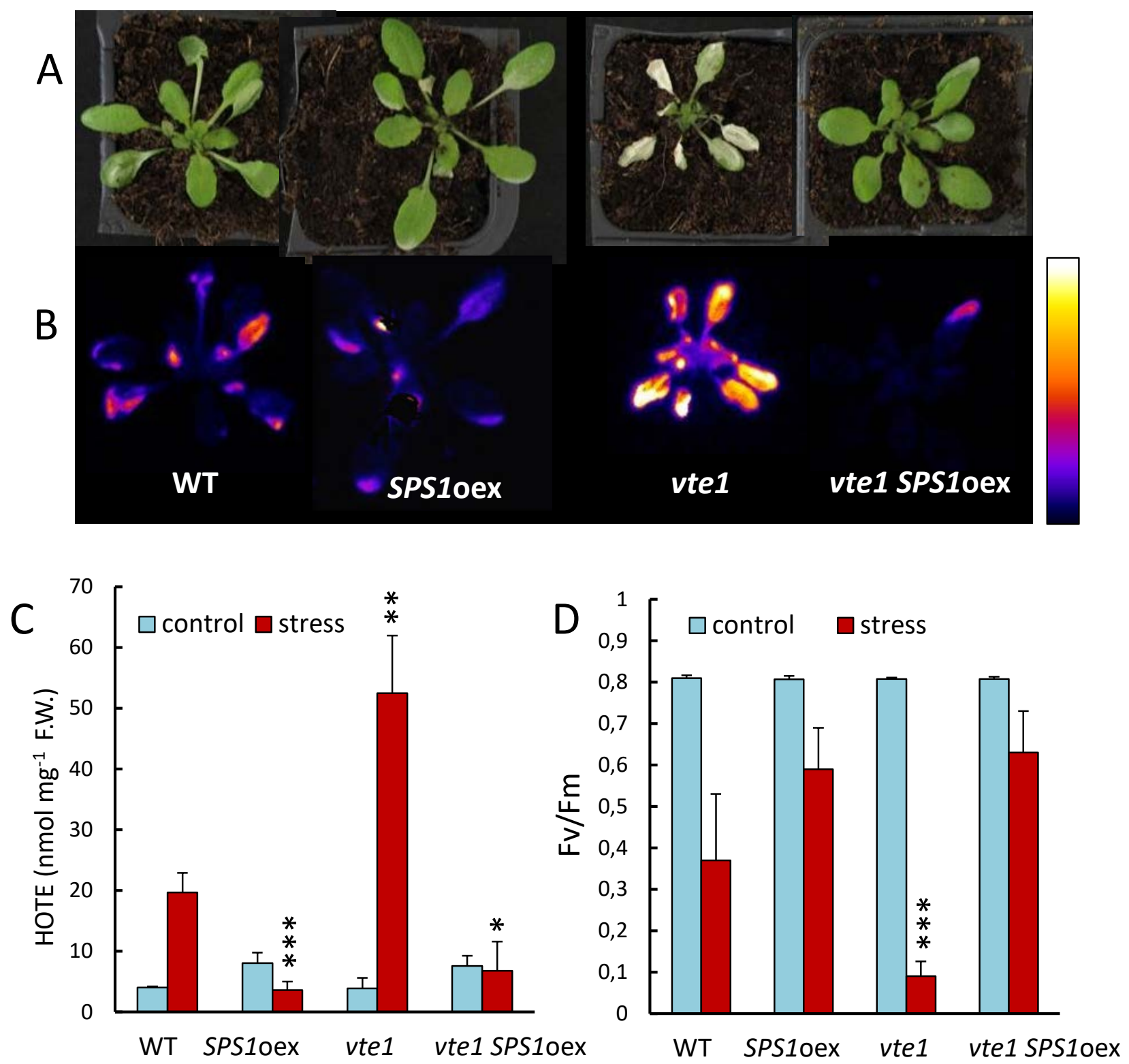

Figure 3 


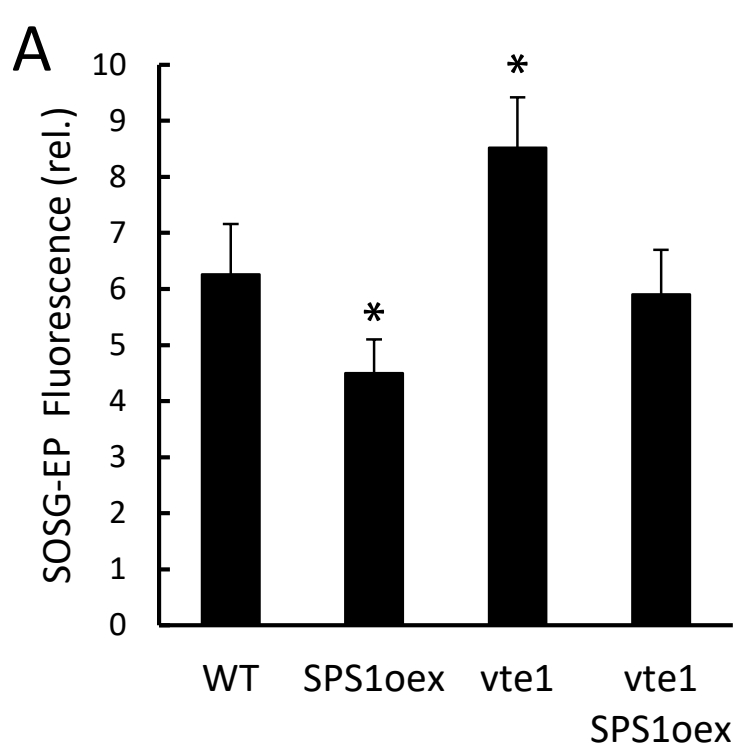

C
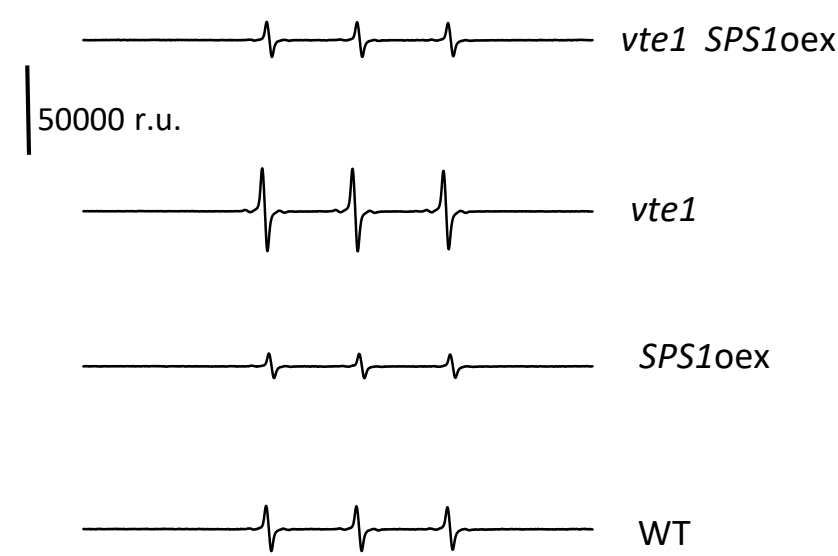

330

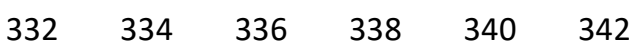

Magnetic field (mT)
B

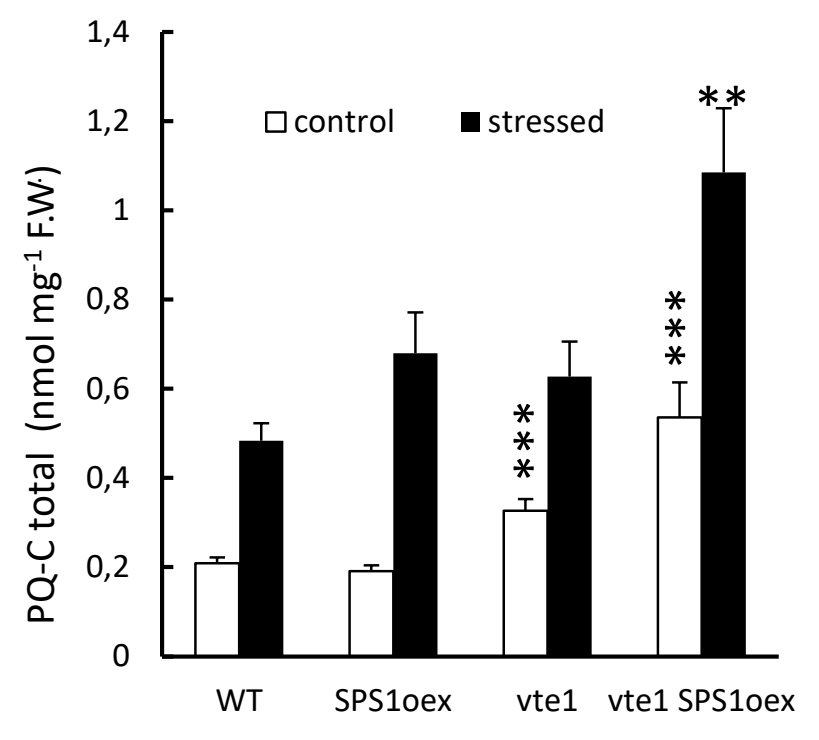

D

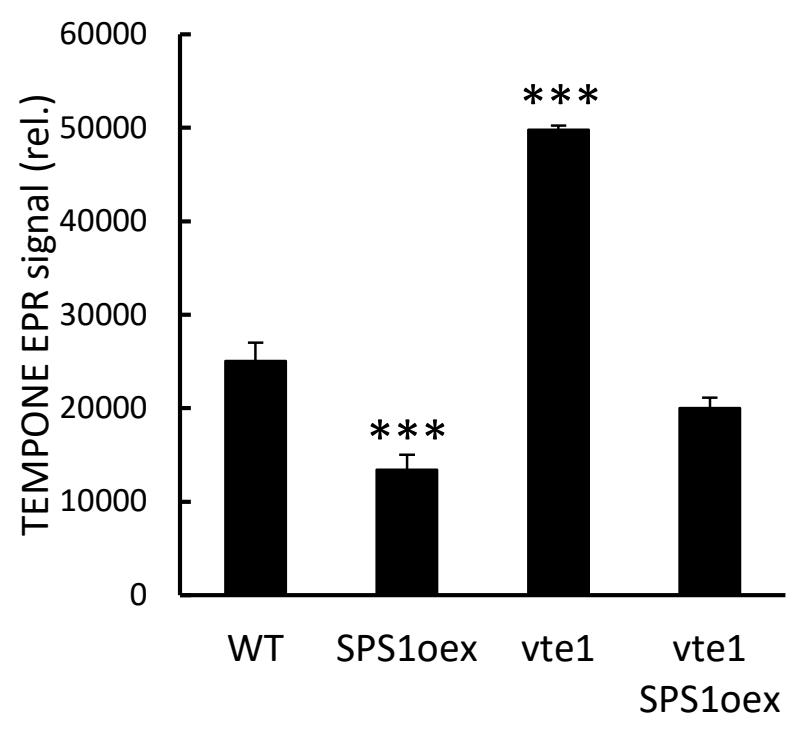

Figure 4 

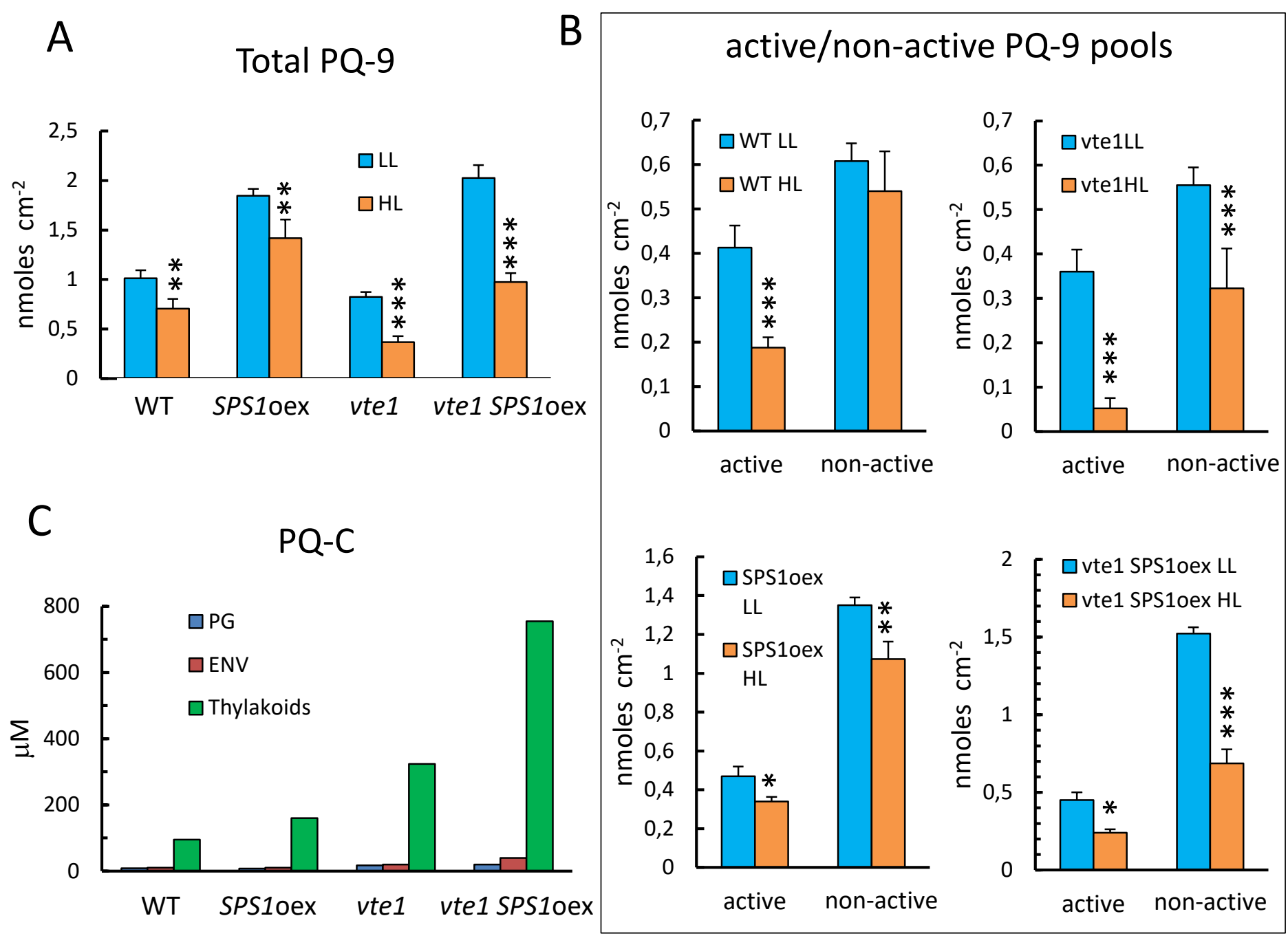

Figure 5 